\title{
Mandibular Advancement Devices for Treating Snoring and Obstructive Sleep Apnea
}

\author{
Jin-Seok Byun, Jae-Kwang Jung \\ Department of Oral Medicine, School of Dentistry, Kyungpook National University, Daegu, Korea
}

Received March 4, 2014

Revised March 12, 2014

Accepted March 31, 2014
Correspondence to:

Jae-Kwang Jung

Department of Oral Medicine, School

of Dentistry, Kyungpook National

University, 2177, Dalgubeol-daero,

Jung-gu, Daegu 700-412, Korea

Tel: +82-53-600-7311

Fax: +82-53-426-2195

E-mail:widenmy@knu.ac.kr
Many therapeutic modalities including continuous positive airway pressure, surgery, and oral appliances are used to treat patients with sleep-disordered breathing. However, there are no definitive treatment modalities for individual patients due to various causes of sleep-disordered breathing. Clinicians should have select best options for individual patients and it is quite challenging process. Oral appliances attracted clinical attention for its convenience and safety. Several designs of oral appliances are introduces such as soft palate lifter, tongue retaining device, and various appliances which aimed to mandibular advancement. Among these oral appliances, mandibular advancement devices (MADs) are considered the most excellent based on their effectiveness and patient tolerance. Although MADs are not guarantee dramatic outcome and less consistent than continuous positive airway pressure, they offer several advantages over continuous positive airway pressure and surgical methods, including non-invasiveness, silence, portability, and tolerability, simplicity. Therefore, general dental practitioner who had passed sleep dental curriculum or coursework can treat the patients with sleep problems. This article reviews the history, clinical indications, suggested mechanism of actions, various positive effects and several side effects, factors predicting a favorable outcome, determining amounts of mandibular advancement, compliance and long-term efficacy of MADs use.

Key Words: Mandibular advancement devices; Sleep apnea syndromes; Snoring

\section{INTRODUCTION}

Sleep-disordered breathing (SDB) is characterized by disturbed breathing during sleep caused by repetitive stenosis and upper airway collapse. ${ }^{1)}$ Vibration in the abundant soft tissues surrounding the airway resulting from the increased airway resistance generates unwanted noise during sleep, known as snoring. Complete upper airway obstruction in severe cases causes recurrent cessation of breathing, referred to as obstructive sleep apnea (OSA). Repetitive airflow reduction or pauses leads to oxygen desaturation and activates the cardiovascular system as well as the central nervous system. Furthermore, the impaired sleep quality secondary to frequent arousal causes excessive daytime sleepiness, mood change, disturbed neurocognition, chronic fatigue, and decreased quality of life (QOL). ${ }^{2)}$ The abnormal cardiovascular activation during sleep is linked with an increased incidence of cardiovascular disease, including hypertension, coronary artery disease, cardiac arrhythmias, and stroke. It also contributes to the increased mortality associated with these diseases in patients with untreated OSA. ${ }^{3)}$ SDB may disrupt intimacy between sleeping partners, increase the incidence of vehicular accidents, cause sexual dysfunction, and aggravate insulin-resistant diabetes mellitus. $^{4,5)}$

SDB treatments comprise professional interventions combined with behavioral modification such as changing the sleep position, avoiding alcohol and sedatives, and weight loss. ${ }^{6}$ Professional interventions include continuous positive airway pressure (CPAP) therapy, surgical reconstruction

Copyright (c) 2014 Korean Academy of Orofacial Pain and Oral Medicine. All rights reserved. 
procedures such as uvulopalatopharyngoplasty (UPPP), and oral appliances. Of these treatment, oral appliances are recently paid attention from many clinicians due to its effectiveness and convenience. ${ }^{7)}$

Oral appliances for treating SDB are classified into mandibular advancement devices (MADs), tongue-retaining devices (TRDs), and soft palatal lifters (SPLs) according to the suggested mechanism of action. ${ }^{8)}$ Among them, MADs are the most frequently used due to their superior efficacy and patient tolerance, and more than 50 designs of MADs have been clinically introduced presently. ${ }^{9,10)}$ In the present review, we summarize the history, mechanisms of action, clinical effects/side effects, determining of mandibular position, patient compliance, and long-term efficacy of MADs.

\section{HISTORY OF MADS AND OTHER ORAL APPLIANCES}

Mandibular advancement appliance, tongue retaining device, and soft palate lifter are representative oral appliances to treat OSA. MADs were first introduced in 1934 by Robin, ${ }^{11)}$ who described a one-piece dental device termed "monobloc" aimed to relieve airway obstruction during sleep in a child patient with retrognathism by repositioning the mandible. However, widespread usage of MADs began much later in the 1980s when MADs were reinvented and the understanding of SDB advanced. In 1985, Soll and George ${ }^{12)}$ introduced modified this "monoblock" to treat severe OSA patient and reported significant decrease in apnea index. Moreover, in 1987, there were another successful results that average apnea index of 44 patients decreased from 50.4 to 23.1 after treatment with prosthetic mandibular advancement. ${ }^{13)}$

Meanwhile, TRDs were first described in 1982 by Cartwright and Samelson, ${ }^{14)}$ and were mainly indicated to the patient who have large tongue or edentulous. SPLs were introduced in 1991 by Paskow and Paskow. ${ }^{15)}$ SPLs are designed to lift the soft palate to reduce the vibration which causes snoring sound.

Numerous subtypes in various designs have since been developed for the three oral appliance types, but TRDs and SPLs were rarely used because of their low patient tolerance and poor efficacy. ${ }^{16)}$ Practically, SPLs did not show any significant reduction in snoring or the apnea-hypopnea index (AHI). ${ }^{17)}$ Even TRDs have significantly less effect on snoring and AHI, and also cause more patient discomfort compared to MADs ${ }^{16)}$; this results in poor tolerability, low compliance, and rejection by patients, although TRDs were as effective as MADs in reducing the AHI in a previous study. ${ }^{18)}$

Barthlen et al. ${ }^{16)}$ observed differing efficacy and tolerance in eight subjects in a comparison of MADs, TRDs, and SPLs. While MADs required at least six teeth per arch to ensure proper retention, TRDs could be used in patients with less than six teeth or even no dentition. ${ }^{16)}$ The study showed that all subjects (100\%) in the MAD group tolerated the appliance, while five subjects in the TRD group and only two subjects in the SPL group tolerated their respective appliances. Furthermore, MADs were significantly more effective than TRDs and SPLs in reducing the AHI. ${ }^{16)}$

\section{CLINICAL INDICATIONS}

Based on American Academy of Dental Sleep Medicine guidelines, MADs are indicated in patients with primary snoring and mild to moderate OSA. ${ }^{19)}$ They are also recommended in patients with severe OSA, and those unable to tolerate $\mathrm{CPAP} .{ }^{20)} \mathrm{MADs}$ are sometimes used as an adjunct to CPAP or other therapies for a better clinical effect. ${ }^{21)}$

According to the guideline of American Sleep Disorders Association, oral appliances are indicated for patients with simple snoring or mild OSA and in patients with moderate to severe OSA who cannot tolerate or refuse CPAP therapy. ${ }^{22)}$ There were reports about the treatment outcomes according to the severities of OSA. Patients with severe OSA had less reduction in apneas (49\%) than patients with mild to moderate OSA (70\%). ${ }^{22,23)}$ In addition, MADs appears to be an effective therapeutic options for treating persistent apneas patients who fail UPPP. ${ }^{24)}$ Twelve of the eighteen patients who failed UPPP were responders to MADs therapy (AHI <10).

Cephalometric studies have been used to predict outcome of MADs treatment. It is generally take for granted that a retrognathic mandible, high position of the hyoid bone, relatively normal post-palatal and post-lingual airway and narrow tongue base could be predictors for successful outcome of MADs therapy. ${ }^{25)}$ 


\section{MECHANISM OF ACTION}

The exact mechanism of action of MADs remains unclear despite several previous studies; however, it is reasonable to conclude that anatomical modification in oropharynx by mandibular advancement provide the favorable circumstance during respiration. ${ }^{26,27)}$ After using MADs, the muscle tone was altered in the masseter, submental, and genioglossus muscles of subjects, muscles likely involved in maintaining the upper airway. ${ }^{28)}$ In addition, the adjacent tissues, including the tongue and hyoid bone, were repositioned while subjects wore MADs. ${ }^{29)}$ The velopharynx and oropharynx were revealed as the primary targeted sites in the upper airway rather than the hypopharynx. ${ }^{30-32)}$ In contrast, a placebo appliance without any protrusive repositioning showed no significant upper airway widening. Therefore, mandibular protrusion is a key factor underlying the clinical mechanism of MADs. Collectively, MADs presumably stabilize and enlarge the pharyngeal airway by repositioning the soft tissue and changing the muscle tone associated with upper airway collapse. ${ }^{33}$

\section{CLINICAL EFFECTS}

The clinical effects of MADs are divided into their direct effects on OSA and indirect effects on the OSA-associated symptoms and complications.

\section{Direct Effects on OSA}

All previous studies showed that MADs significantly reduced objective respiratory indices such as AHI and respiratory disturbance index (RDI) compared to placebo or baseline. ${ }^{34)}$ Despite this clear outcome, previous studies also found that the reductions in respiratory indices with MADs were both less effective and more variable than those achieved with CPAP. ${ }^{34)}$ Previous studies reported that MADs decreased the mean AHI or RDI to 4.5-34, corresponding to a mean 28\%-80\% reduction rate (overall mean reduction rate of 55\%). ${ }^{33,34)}$ In contrast, CPAP reduced these indices from a mean of $18-40$ to $2.4-8.0$, corresponding to a $74 \%$ 94\% mean reduction rate (overall $83 \%$ mean reduction). ${ }^{34)}$ Therefore, to improve the clinical predictability, a careful approach is needed and the procedure should be performed by qualified dental specialists. ${ }^{19)}$

\section{Indirect Effects on OSA-Associated Symptoms or Complications}

Contrary to the effects on AHI or RDI, no previous study reports any significance on OSA-associated symptoms or complications when compared to a placebo or even baseline. However, despite these conflicting and diverse results, many previous reviews suggest that MADs may significantly affect OSA-associated symptoms and complications.

\section{1) Sleepiness}

Effects on subjective daytime sleepiness have been mostly evaluated using the Epworth sleepiness scale (ESS). Several placebo-controlled studies revealed that subjective sleepiness decreased to almost normal or by 1-4 points after implementing MADs. ${ }^{35-38)}$ The relief of SDB improved the sleep quality, manifested in the reduced mean arousal index, which is notable as sleep quality is believed to play an important role in reducing daytime sleepiness. ${ }^{35)}$ However, improved daytime sleepiness was sometimes observed without a concurrent normalization of objective respiratory indices. ${ }^{35,39)}$ A previous study on the effects of MADs found that the ESS scores normalized in $82 \%$ of subjects, whereas snoring was completely relieved in only $23 \%$ of subjects. ${ }^{35}$ These findings indicate that factors other than the objective respiratory indices also contribute to excessive daytime sleepiness in patients with SDB. MADs are believed to improve subjective sleepiness by altering unknown factors as well as improving respiratory parameters. MADs are similar to or less effective than CPAP in reducing daytime sleepiness. ${ }^{40)}$

Objective sleepiness has been examined using the multiple sleep latency test (MSLT), Osler test, and the maintenance of wakefulness test (MWT). A controlled study evaluating MSLT found improved objective sleepiness compared to the placebo. ${ }^{35)}$ In addition, two studies using the Osler test or MWT reported similar results ${ }^{40,41}$; otherwise, a different study reported no significant improvement in MWT. ${ }^{42)}$ MADs are considered to have a similar or lower effect on objective sleepiness in comparison with $\mathrm{CPAP}^{43)}$ 


\section{2) Snoring}

Snoring itself is frequently major reason why the patients come to the clinics. Non-apneic snorer without daytime sleepiness do usually not tolerate to the CPAP therapy. Even though they agree easily and try to use the CPAP, but considerable patients will stop using the CPAP before long. Surgical interventions are also risky to the patients of simple snoring without apnea and often cause resistance to patients due to its irreversible results. Patients who prefer noninvasive and simple methods usually select oral appliance options as first choice to solve their snoring.

Of the various oral appliances, SPL was originally invented to reduce vibration of soft tissue, however, it turned out that there is no advantages in using SPL to treat snoring. TRDs could be another option for treating the snoring. According to George ${ }^{44)}$ snoring was decreased or completely disappeared after TRDs use. Other reports also support the effectiveness of TRDs to snoring patients in an aspect of decreased visual analogue scale and percent of loud snoring time. ${ }^{45,46)}$ Despite of its effectiveness on snoring, TRDs have lower tolerance to the patient due to the associated adverse effects. ${ }^{45)}$

MADs are the most popular and appropriate oral appliances to treat snoring and more than 30 articles reported the effectiveness of MADs. Most of investigations concluded that MADs are beneficial in reducing snoring, and majority of the randomized, controlled studies found significant reduction of snoring. In detail, MADs significantly reduce snoring; the frequency of snoring was reportedly decreased between 13\%-90\% in numerous studies. By evaluating the objective and subjective results collectively, the severity of snoring is reportedly reduced by $45 \% .^{33)}$ Another study reported that the severity and intensity of snoring were reduced by $47 \%$ and $3 \mathrm{~dB}$, respectively. ${ }^{47)}$ Because decreased snoring was linked with improved OSA, persistent snoring may imply insufficient clinical effect of MADs on OSA, indicating the need for further treatment. ${ }^{48)}$ Though the CPAP is known to reduce snoring more than MADs, but regarding the cost/benefit effects and compliances, MADs are generally preferred than CPAP to both patients and clinicians. ${ }^{49)}$

\section{3) Neurocognition}

One of main social concern of OSA is increased risk of accidents due to the dampened recognition while daytime activities. Regarding to the degree of arousal, there are several reports described the correlation between using MADs and neuro-responsiveness. A previous study reported that the use of MADs for 2-3 months improved the simulated driving performance comparable to improvements observed with $\mathrm{CPAP}^{43)}$ While improved neurocognition and decreased daytime sleepiness are expected to decrease the risk of vehicular accidents, there is no study confirming whether MADs usage actually prevents vehicular accidents. According to Naismith et al., ${ }^{37)} 4$ weeks use of MADs improved self-reported sleepiness, fatigue/energy levels, however, the level of attention/memory, visuospatial or executive functioning were not improved. While several studies were performed to evaluate neurocognition improvements in children/adolescents after surgery or CPAP. ${ }^{50,51)}$ The exact effects on neurocognition in children/adolescents remain to be clearly established.

\section{4) Quality of life}

Evaluating the QOL is quit challenging because it is dependent on individuals and not easy to objectify. However, it is valuable to measure patient's subjective symptoms not only objective variables in a view of clinical failure or success. To study the relation between QOL and sleep problem, researchers usually depend on various questionnaire. Previous studies have evaluated the effects of MADs on QOL using various tools, including the Minor Symptoms Evaluation-Profile (MSE-P), 36-Item Short-Form Health Survey (SF-36), and Calgary Sleep Apnea Quality of Life Index (SAQLI), and showed improvement in several variables compared to a placebo. ${ }^{39,42)}$ However, Barnes et al. ${ }^{42)}$ found no significant improvement in the SF-36, Beck Depression Index, or Profile of Mood States Total Mood Disorder scores compared to the placebo tablet, except in the Functional Outcomes of Sleep Questionnaire (FOSQ). Lam et al. ${ }^{39)}$ reported improved QOL based on the SAQLI, which indicates that MADs were more effective than CPAPs. Another study reported significant improvements in mean vitality, contentment, and sleep in patients using MADs similar to those undergoing UPPP after 1 year of treatment. ${ }^{52)}$ In addition, the effects of MADs were similar or better than those of CPAP. One important considering thing when comparing self-reported QOL from different studies 
is variable criteria and medical conditions of individual patient. These factors can significantly affect the subjective symptoms, interpreters and researches have to keep in mind these limitation or bias.

\section{5) Cardiovascular symptoms}

There were numerous investigations to verify the relationship between sleep apnea and cardiovascular problems, such as hypertensions, coronary artery disease, and cerebrovascular disease. Because the application of oral appliance can indicated to moderate OSA, there have been several reports to examine the effectiveness of MADs on cardiovascular symptoms in OSA patient. Significant reduction in some variables of blood pressure (BP) has been reported after treatment using MADs. ${ }^{39,42)}$ In patients with an AHI $>10$, after 4 weeks of using MADs, the 24-hour diastolic BP decreased by $2 \mathrm{mmHg}$ and $\mathrm{AHI}$ decreased compared to the placebo. ${ }^{53)}$ Another study showed a 3-mmHg BP decrease during wakefulness, but not during sleep. Yet another study observed a significant decrease in nocturnal diastolic BP but not in the 24-hour BP after MAD use for 3 months. ${ }^{42]}$ Lam et al. ${ }^{54)}$ reported that the significantly reduced systolic $\mathrm{BP}$ associated with MADs was maintained during a 1-year follow-up. In addition, two studies showed a significantly reduced single $\mathrm{BP}$, although the measurement method used in that study was less reliable. ${ }^{39,55)}$

Endothelial reactivity is also likely to improve after using MADs. ${ }^{34)}$ A previous study showed similar improvements in microvascular reactivity after treatment with either MADs or CPAPs and suggested that the increased vasodilation may reflect decreased nocturnal oxygen desaturation. ${ }^{56)}$ More interestingly, after 1 year of MAD use, the improved endothelial function approached that of normal subjects. ${ }^{57)}$ Finally, Anandam et al ${ }^{58)}$ showed in a controlled cohort study that the cardiovascular death rate was significantly and equally lower in MAD or CPAP users than in non-users, although AHI was significantly higher in MAD users than among CPAP users.

\section{CONSIDERATION OF PREVIOUS SUCCESS CRITERIA}

Previous studies have assessed the treatment outcome using variable measures including objective respiratory indices (AHI or RDI, snoring frequency, oxygen desaturation), subjective symptom questionnaires (daytime sleepiness, QOL, sexual dysfunction), and objective symptom measurements (BP, endothelial reactivity). The criteria of success were exclusively based on AHI or RDI, while other variables were used as supportive indices. Alternatively, full polysomnogram (PSG) is the gold standard diagnostic method with excellent accuracy and reliability. Many studies defined successful treatment as an AHI $<5$ or AHI $<10 .{ }^{34)}$ Considering that the normal range is $<5$, an AHI $<5$ may be the more reasonable objective criteria of successful treatment.

Despite the strict success criteria in treating OSA, MADs are recently selected "first" option in treating mild to moderate OSA, even in some severe cases. Although MADs treatment does not show constant and predictable outcomes described above compare to CPAPs, it is valuable to try the MADs in consideration of patient's compliance and costeffective benefits. Therefore the success criteria for MADs need to be little bit broad and less particular than other therapeutic options.

\section{FACTORS PREDICTING A FAVORABLE OUTCOME}

Greater variability in treatment outcomes was observed with MADs than with CPAP; therefore, patient selection is more important in improving the predictability of MAD treatment. ${ }^{34)}$ Accordingly, several studies have attempted to identify factors predicting a better MAD treatment outcomes. Studies suggested several variables, including age, gender, obesity, sleep posture dependency, collapse site, and OSA severity as predictive factors. Among patients with OSA, MADs were more likely to be effective in patients that were female, younger, and less obese. ${ }^{59,60)}$ Patients with mild, moderate, or positional OSA also seem to respond better to MADs. ${ }^{36,61)}$ Several studies measuring the airway space characterize the diagnostic utility of cephalography, computed tomography, magnetic resonance imaging (MRI), and other imaging techniques.

\section{OSA Severity}

MADs is likely to be more effective in patients with mild 
OSA and more frequently achieves normalization in patients with mild and moderate OSA than in those with severe OSA. ${ }^{36,61)}$ A study found that 21 of 25 patients with mild to moderate OSA normalized, while only 8 of 26 patients with severe OSA normalized when using MADs. ${ }^{61)}$ Another study reported that the rate of decrease to an AHI $<10$ after using MADs was 81\%, 60\%, and 25\% in patients with mild, moderate, and severe 0SA, respectively. ${ }^{36,24)}$ However, although post-treatment AHI in most patients remained above a normal AHI, this study also found that the magnitude of the AHI decrease was greater in patients with severe OSA, from 53 to $14{ }^{24)}$ These findings were supported by similar findings in a relatively large study evaluating the PSG in 397 patients. ${ }^{36)}$ Both studies showed that the magnitude of the AHI decrease was significantly decreased in the severe OSA group, and normalization rate was decreased in both the moderate and severe OSA groups using MADs. ${ }^{36}$ These findings indicate the superior outcome of CPAP compared to MADs in decreasing AHI.

\section{Age, Gender, Obesity, and Sleep Posture Dependency}

MADs are more likely to be effective in patients that are female, younger, and less obese ${ }^{59,60)}$; they are also more efficacious in patients with supine position-dependent OSA. ${ }^{62,63)}$ Liu et al. ${ }^{60)}$ showed that in subjects with a good, moderate, or poor AHI decrease, the mean body mass index (BMI) was significantly lower in those with a good outcome (mean BMI=27.26) than in those with a poor outcome (mean BMI=32.14). Previous studies suggested that excess tissues surrounding the upper airway in obese patients promoted airway collapse. In particular, truncal obesity altered the breathing pattern and reduced oxygen storage, thereby compromising effective muscle tone activation associated with mandibular advancement. ${ }^{64)}$ The better response in female patients may reflect their comparatively greater pharyngeal enlargement during mandibular advancement, as well as their stiffer and less collapsible airway. ${ }^{63,65,66)}$ Treatment outcome disparities associated with age may be explained by the decreased ability of respiratory muscles to generate tension and resist fatigue, increasing the ventilatory instability during sleep. ${ }^{60,67)}$ In a study of posturedependent OSA, Chung et al. ${ }^{62)}$ reported that MADs reduced AHI by $74.69 \%$ in patients with positional OSA, but only
46.03\% in patients with non-positional OSA. Their study also found that the positional OSA group had a higher BMI than the non-positional group did, and they surmised that the non-positional OSA patients may have a more collapsible pharyngeal airway that is possibly worsened by obesity. ${ }^{62)}$

\section{Anatomic Variables}

Many studies have compared the anatomic differences between good and poor responders and suggest several predictive factors. Several studies have particularly focused on the efficacy of MADs according to the upper airway collapse site. MADs were reportedly more effective in OSA patients with collapse localized in the oropharynx rather than the velopharynx. ${ }^{31,32)}$ However, the anatomic site most likely to respond to MADs has been localized to different upper airway region. A previous study found that in good responders, a larger cross-sectional area of the velopharynx was involved than that observed in poor responders. ${ }^{30)}$ Another study using MRI found a similar result. ${ }^{68)}$ Therefore, despite other similar studies reporting that the hypopharynx was likely to respond MADs, the ideal anatomic site is undetermined. In addition, certain cephalometric variables appear to affect the treatment outcome. Among them, a higher positioned hyoid bone and smaller upper airway space were closely associated with a better response to MADs. ${ }^{60)}$

\section{SIDE EFFECTS}

Side effects caused by MADs are relatively frequent but mild in intensity. ${ }^{35)}$ Side effects occurred more frequently in subjects treated with MADs than in those treated with a placebo. ${ }^{35)}$ Typical immediate side effects include jaw discomfort, tooth tenderness, dry mouth, and excessive salivation immediately after using MADs; long-term effects include tooth movement, dry mouth, and jaw discomfort. ${ }^{30}{ }^{3}$ Specifically in patients experiencing occlusal alterations, previous studies reported a decreased overjet and overbite. After an average 7.3 years of using MADs, Almeida et al. ${ }^{69)}$ reported mean overbite and overjet reductions of 1.9 $\mathrm{mm}$ and $1.2 \mathrm{~mm}$, respectively. Similarly, Marklund ${ }^{70)}$ found that both overbite and overjet decreased by $0.6 \mathrm{~mm}$ over a 5-year follow-up of 155 patients with OSA. However, 
there was no alteration in the condylar shape after using MADs for 1 year. ${ }^{71)}$ These findings suggest that the occlusal change was caused by teeth migration rather than condylar change. Furthermore, these occlusal alterations were considered mild and favorable and are not generally linked to disturbed mastication. ${ }^{72)}$ Other side effects were usually relieved by symptomatic treatment, temporary cessation and, if needed, further adjustment of the device. The frequency of side effects associated with MADs is similar to that associated with CPAP. $^{40)}$ Therefore, periodic follow-up is needed to manage potential side effects or discomfort.

\section{DETERMINING THE THERAPEUTIC POSITION OF THE MANDIBLE}

The mandibular position is directed forward and downward in patients using MADs. Increasing the mouth opening from 4 to $14 \mathrm{~mm}$ did not significantly affect the $\mathrm{AHI}^{3{ }^{73}}$ Contrary to this finding, another study showed that increasing the vertical dimension (VD) without mandibular protrusion aggravated AHI in approximately $50 \%$ of subjects. ${ }^{74)}$ In addition, increased VD enhanced temporomandibular symptoms and decreased patient preference. ${ }^{73)}$ Therefore, unnecessary increases in VD are not recommended.

While the optimal mandibular protrusion for managing OSA and snoring depends on the individual patient, mandibular protrusion appears to have a dose-dependent nonlinear association with decreasing $\mathrm{AHI}$ and increasing frequency of side effects. ${ }^{75)}$ Reportedly, patients with 50\%-75\% of maximum mandibular protrusion had a more than 25\% AHI decrease. ${ }^{75,76)}$ However, the side effect prevalence increased significantly beyond 50\% of maximum mandibular protrusion. ${ }^{76)}$ Therefore, the beginning at $50 \%$ of maximum mandibular protrusion may be best, though this was not confirmed. In addition, the protrusion range had less effect on patients with mild and moderate than those with severe OSA. $^{77)}$

\section{COMPARISON BETWEEN DIFFERENT TYPES OF MADS}

There are a few studies comparing different MADs. Nonadjustable MADs were considered less practical because the appliance must be sectioned when further adjustment is needed to improve the efficacy or convenience. ${ }^{78)}$ Regarding the durability, fractures occurred in $60 \%$ of adjustable MADs with a Herbst joint within 3.6 years ${ }^{79}$; a 5-year follow-up study reported that nonadjustable devices required replacement in 6 of 19 patients (approximately 31.6\%). ${ }^{80)}$ One previous study reported better clinical effect using the non-adjustable MADs than the adjustable MADs, but other studies reported less AHI improvements with non-adjustable MAD than adjustable MAD. ${ }^{81,82)}$

In a comparison of two different adjustable MADs, the two devices had similar clinical effects after 2 years of use. ${ }^{83)}$ Therefore, criteria for selecting a specific MAD model should be determined based on practicality, feasibility, and convenience. Thermoplastic non-customized MADs did not effectively decrease AHI and only decreased snoring to a limited extent. ${ }^{84)}$ This minimal therapeutic effect reflects the inability of the unit to maintain appropriate therapeutic protrusive mandibular position, causing poor retention. Poor retention of thermoplastic non-customized MADs was also likely caused by a decrease in patient preference following the increased discomfort. ${ }^{84)}$ Therefore, thermoplastic non-customized MADs are not recommended for treating SDB or even for preliminary MADs evaluation. ${ }^{34}$

\section{PREFERENCE AND COMPLIANCE}

MADs are strongly preferred to CPAP or placebo appliances by most of patients. ${ }^{48}$ A previous study found that $81 \%$ of patients selected MADs, whereas only 19\% selected CPAP ${ }^{85)}$ The high preference is another advantage of MADs, which were portable, silent, and better tolerated than CPAP therapy, and more effective than a placebo appliances. Contrary to most previous studies, one study reported a slightly higher preference for CPAP than MADs. ${ }^{42)}$ In this randomized controlled crossover trial of 3 months of treatment, post-treatment AHI was significantly lower in patients using CPAP than in those using MADs. ${ }^{42)}$ Therefore, the patient preference appeared to depend on not only comfort but also effectiveness. ${ }^{86)}$ Based on self-reports by patients, short-term compliance reached $76 \%-95 \%$, which is higher than that observed in CPAP. ${ }^{34)}$ However, compliance decreased over time, declining from $76 \%$ in the first year to 
approximately 50\% in the second to fourth years. ${ }^{34,83)}$

One review article revealed that despite the high initial compliance, the compliance of MADs was likely to decrease more sharply than CPAP use, approaching a similar compliance to that of long-term use of CPAP. ${ }^{87)}$ Patients who ceases the MADs mostly pointed out its ineffectiveness and discomfort. Therefore, the clinician must have reevaluate the condition of MADs to maintain compliances, including routine examinations and adjustment, along with repetitive patient education. $^{49,83)}$

\section{LONG-TERM EFFICACY}

Because of OSA treatment is a lifelong process, long-term follow-up is important when it comes to evaluating success or failures of treatment and compliance. However, there are few long-term follow-up studies to evaluate the efficacy of treatment. Previous studies found that the initial drop in AHI with MADs was usually maintained or slightly increased over time. Another study showed that MADs were still able to effectively decrease AHI after 2 years despite an increased subjective daytime sleepiness. ${ }^{83)}$ Increased subjective sleepiness may reflect the tendency of patients to take initial improvements in daytime sleepiness for granted as time advances. ${ }^{83)}$ As oral appliances are applied to more OSA patients, the need of long-term efficacy data will be growing.

\section{CONCLUSIONS}

MADs are slightly better in reducing AHI than UPPP, but less effective than CPAP therapy. The effectiveness tend to last for a relatively long period of years compared to UPPP, but subjective sleepiness increased with time. MADs may improve quality as well as quantity of life by alleviating related symptoms and reduce potential serious complications.

MADs showed more variable outcomes compared to CPAP therapy, therefore, appropriate patient selection is more important to improve the clinical predictability. Though the initial patient preference and compliance for MADs is high, these parameters are more likely to decrease with time than in CPAP. Patient's poor compliance is likely caused by uncontrolled side effects and ineffectiveness.

The frequency of side effects is reportedly lower in MADs than in UPPP and similar to CPAP. Furthermore, their severity is mild and transient in almost all cases. Optimal mandibular positioning for MADs is essential to maximize the efficacy and minimize side effects, which ultimately
1. Do you know history of various oral appliance and backgrounds?

2. Can you select the patient who are expected to show favorable outcomes with oral appliances?

3. Do you know the suggested mechanism of action of oral appliance, especially MADs?

4. Do you know various clinical effects after MADs use? (e.g., AHI/RDI, sleepiness, snoring, QOLs, cardiovascular problem)

5. Can you explain consideration of success criteria for MADs use?

6. Do you know factors predicting favorable outcomes of MADs?

7. Do you know the therapeutic mandibular position and have ability to manage the side effect during the treatment?

8. Do you understand long-term efficacy of MADs compare to other treatment such as CPAP and surgery?
Fig. 1. Preliminary check lists for dental practitioners in treating obstructive sleep apnea/snoring patient with mandibular advancement devices (MADs). AHI, apneahypopnea index; RDI, respiratory disturbance index; QOLs, quality of lifes; CPAP, continuous positive airway pressure. 
increases the long-term compliance. Clinicians should attempt to find the optimal mandibular position in their patients, which may require additional adjustment and evaluation. For this reason, treatment using MADs should be primarily supervised by qualified dental specialists who are able to maintain effective function of the device. However, MADs are basically safe and easily to access than other treatment options, even general dental practitioner who had passed sleep dental curriculum or seminar can treat the patients with mild to moderate OSA and snoring patient. General dental practitioners who want to treat the patient with sleep problem with oral appliances have to diagnose properly and have the abilities to solve simple adverse effects during the treatment. We suggest Fig. 1 which is the check lists of minimum requirement of knowledge to treat the sleep-disturbed breathing patient with MADs. MADs are useful treatment option for OSA and snoring, and dentists have to actively participate to improve their QOLs.

\section{CONFLICT OF INTEREST}

No potential conflict of interest relevant to this article was reported.

\section{REFERENCES}

1. Guilleminault C, Tilkian A, Dement WC. The sleep apnea syndromes. Annu Rev Med 1976;27:465-484.

2. White DP. Sleep apnea. Proc Am Thorac Soc 2006;3:124-128.

3. Dasheiff RM, Finn R. Clinical foundation for efficient treatment of obstructive sleep apnea. J Oral Maxillofac Surg 2009;67:21712182.

4. Harding SM. Complications and consequences of obstructive sleep apnea. Curr Opin Pulm Med 2000;6:485-489.

5. Terán-Santos J, Jiménez-Gómez A, Cordero-Guevara J. The association between sleep apnea and the risk of traffic accidents. Cooperative Group Burgos-Santander. N Engl J Med 1999;340:847851.

6. Morgenthaler TI, Kapen S, Lee-Chiong T, et al; Standards of Practice Committee; American Academy of Sleep Medicine. Practice parameters for the medical therapy of obstructive sleep apnea. Sleep 2006;29:1031-1035.

7. Epstein LJ, Kristo D, Strollo PJ Jr, et al; Adult Obstructive Sleep Apnea Task Force of the American Academy of Sleep Medicine. Clinical guideline for the evaluation, management and longterm care of obstructive sleep apnea in adults. J Clin Sleep Med 2009;5:263-276.

8. Strauss AM. Oral devices for the management of snoring and obstructive sleep apnea. In: Fairbanks DNF, Fujita R, eds. Snoring and obstructive sleep apnea. 2nd ed. New York: Raven Press Ltd.; 1994. pp. 229-241.

9. Clark GT. Mandibular advancement devices and sleep disordered breathing. Sleep Med Rev 1998;2:163-174.

10. Lowe AA, Sjöholm TT, Ryan CF, Fleetham JA, Ferguson KA, Remmers JE. Treatment, airway and compliance effects of a titratable oral appliance. Sleep 2000;23(Suppl 4):S172-S178.

11. Robin P. Glossoptosis due to atresia and hypotrophy of the mandible. Arch Pediatr Adolesc Med 1934;48:541-547.

12. Soll BA, George PT. Treatment of obstructive sleep apnea with a nocturnal airway-patency appliance. N Engl J Med 1985;313: 386-387.

13. Meier-Ewert K, Brosig B. Treatment of sleep apnea by prosthetic mandibular advancement. In: Peter JH, Podszus T, von Wichert P, eds. Sleep related disorders and internal diseases. Heidelberg: Springer Verlag GmbH; 1987. pp. 341-345.

14. Cartwright RD, Samelson CF. The effects of a nonsurgical treatment for obstructive sleep apnea. The tongue-retaining device. JAMA 1982;248:705-709.

15. Paskow H, Paskow S. Dentistry's role in treating sleep apnea and snoring. N J Med 1991;88:815-817.

16. Barthlen GM, Brown LK, Wiland MR, Sadeh JS, Patwari J, Zimmerman M. Comparison of three oral appliances for treatment of severe obstructive sleep apnea syndrome. Sleep Med 2000;1:299305.

17. Marklund M, Franklin KA. Dental appliances in the treatment of snoring. A comparison between an activator, a soft-palate lifter, and a mouth-shield. Swed Dent J 1996;20:183-188.

18. Deane SA, Cistulli PA, Ng AT, Zeng B, Petocz P, Darendeliler MA. Comparison of mandibular advancement splint and tongue stabilizing device in obstructive sleep apnea: a randomized controlled trial. Sleep 2009;32:648-653.

19. Kushida CA, Morgenthaler TI, Littner MR, et al; American Academy of Sleep. Practice parameters for the treatment of snoring and obstructive sleep apnea with oral appliances: an update for 2005. Sleep 2006;29:240-243.

20. Lee CH, Mo JH, Choi IJ, et al. The mandibular advancement device and patient selection in the treatment of obstructive sleep apnea. Arch Otolaryngol Head Neck Surg 2009;135:439-444.

21. Borel JC, Gakwaya S, Masse JF, Melo-Silva CA, Sériès F. Impact of CPAP interface and mandibular advancement device on upper airway mechanical properties assessed with phrenic nerve stimulation in sleep apnea patients. Respir Physiol Neurobiol 2012;183:170-176.

22. Pancer J, Al-Faifi S, Al-Faifi M, Hoffstein V. Evaluation of variable mandibular advancement appliance for treatment of snoring and sleep apnea. Chest 1999;116:1511-1518.

23. Marklund M, Franklin KA, Sahlin C, Lundgren R. The effect of a mandibular advancement device on apneas and sleep in patients with obstructive sleep apnea. Chest 1998;113:707-713.

24. Millman RP, Rosenberg CL, Carlisle CC, Kramer NR, Kahn DM, Bonitati AE. The efficacy of oral appliances in the treatment of persistent sleep apnea after uvulopalatopharyngoplasty. Chest 1998;113:992-996.

25. Mayer G, Meier-Ewert K. Cephalometric predictors for orthopae- 
dic mandibular advancement in obstructive sleep apnoea. Eur J Orthod 1995;17:35-43.

26. L'Estrange PR, Battagel JM, Harkness B, Spratley MH, Nolan PJ, Jorgensen GI. A method of studying adaptive changes of the oropharynx to variation in mandibular position in patients with obstructive sleep apnoea. J Oral Rehabil 1996;23:699-711.

27. Sam K, Lam B, Ooi CG, Cooke M, Ip MS. Effect of a non-adjustable oral appliance on upper airway morphology in obstructive sleep apnoea. Respir Med 2006;100:897-902.

28. Kurtulmus H, Cotert S, Bilgen C, On AY, Boyacioglu H. The effect of a mandibular advancement splint on electromyographic activity of the submental and masseter muscles in patients with obstructive sleep apnea. Int J Prosthodont 2009;22:586-593.

29. Almeida FR, Tsuiki S, Hattori Y, Takei Y, Inoue Y, Lowe AA. Dose-dependent effects of mandibular protrusion on genioglossus activity in sleep apnoea. Eur Respir J 2011;37:209-212.

30. Chan AS, Lee RW, Srinivasan VK, Darendeliler MA, Grunstein RR, Cistulli PA. Nasopharyngoscopic evaluation of oral appliance therapy for obstructive sleep apnoea. Eur Respir J 2010;35:836842.

31. Choi JK, Hur YK, Lee JM, Clark GT. Effects of mandibular advancement on upper airway dimension and collapsibility in patients with obstructive sleep apnea using dynamic upper airway imaging during sleep. Oral Surg Oral Med Oral Pathol Oral Radiol Endod 2010;109:712-719.

32. Ng AT, Qian J, Cistulli PA. Oropharyngeal collapse predicts treatment response with oral appliance therapy in obstructive sleep apnea. Sleep 2006;29:666-671.

33. Hoffstein V. Review of oral appliances for treatment of sleepdisordered breathing. Sleep Breath 2007;11:1-22.

34. Marklund M, Verbraecken J, Randerath W. Non-CPAP therapies in obstructive sleep apnoea: mandibular advancement device therapy. Eur Respir J 2012;39:1241-1247.

35. Gotsopoulos H, Chen C, Qian J, Cistulli PA. Oral appliance therapy improves symptoms in obstructive sleep apnea: a randomized, controlled trial. Am J Respir Crit Care Med 2002;166:743-748.

36. Holley AB, Lettieri CJ, Shah AA. Efficacy of an adjustable oral appliance and comparison with continuous positive airway pressure for the treatment of obstructive sleep apnea syndrome. Chest 2011;140:1511-1516.

37. Naismith SL, Winter VR, Hickie IB, Cistulli PA. Effect of oral appliance therapy on neurobehavioral functioning in obstructive sleep apnea: a randomized controlled trial. J Clin Sleep Med 2005;1:374-380.

38. Maguire J, Steele JG, Gibson GJ, Wilson JA, Steen N, McCracken GI. Randomised cross-over study of oral appliances for snoring. Clin Otolaryngol 2010;35:204-209.

39. Lam B, Sam K, Mok WY, et al. Randomised study of three nonsurgical treatments in mild to moderate obstructive sleep apnoea. Thorax 2007;62:354-359.

40. Engleman HM, McDonald JP, Graham D, et al. Randomized crossover trial of two treatments for sleep apnea/hypopnea syndrome: continuous positive airway pressure and mandibular repositioning splint. Am J Respir Crit Care Med 2002;166:855-859.

41. Gagnadoux F, Fleury B, Vielle B, et al. Titrated mandibular advancement versus positive airway pressure for sleep apnoea. Eur
Respir J 2009;34:914-920.

42. Barnes M, McEvoy RD, Banks S, et al. Efficacy of positive airway pressure and oral appliance in mild to moderate obstructive sleep apnea. Am J Respir Crit Care Med 2004;170:656-664.

43. Hoekema A, Stegenga B, Bakker M, et al. Simulated driving in obstructive sleep apnoea-hypopnoea; effects of oral appliances and continuous positive airway pressure. Sleep Breath 2007;11:129-138.

44. George PT. A modified functional appliance for treatment of obstructive sleep apnea. J Clin Orthod 1987;21:171-175.

45. Cartwright R, Venkatesan TK, Caldarelli D, Diaz F. Treatments for snoring: a comparison of somnoplasty and an oral appliance. Laryngoscope 2000;110:1680-1683.

46. Schönhofer B, Stoohs RA, Rager H, Wenzel M, Wenzel G, Köhler D. A new tongue advancement technique for sleep-disordered breathing: side effects and efficacy. Am J Respir Crit Care Med 1997;155:732-738.

47. Mehta A, Qian J, Petocz P, Darendeliler MA, Cistulli PA. A randomized, controlled study of a mandibular advancement splint for obstructive sleep apnea. Am J Respir Crit Care Med 2001;163: 1457-1461.

48. Ferguson KA, Ono T, Lowe AA, Keenan SP, Fleetham JA. A randomized crossover study of an oral appliance vs nasal-continuous positive airway pressure in the treatment of mild-moderate obstructive sleep apnea. Chest 1996;109:1269-1275.

49. Aarab G, Lobbezoo F, Hamburger HL, Naeije M. Oral appliance therapy versus nasal continuous positive airway pressure in obstructive sleep apnea: a randomized, placebo-controlled trial. Respiration 2011;81:411-419.

50. Biggs SN, Vlahandonis A, Anderson V, et al. Long-term changes in neurocognition and behavior following treatment of sleep disordered breathing in school-aged children. Sleep 2014;37:77-84.

51. Kennedy JD, Blunden S, Hirte C, et al. Reduced neurocognition in children who snore. Pediatr Pulmonol 2004;37:330-337.

52. Walker-Engström ML, Wilhelmsson B, Tegelberg A, Dimenäs E, Ringqvist I. Quality of life assessment of treatment with dental appliance or UPPP in patients with mild to moderate obstructive sleep apnoea. A prospective randomized 1-year follow-up study. J Sleep Res 2000;9:303-308.

53. Gotsopoulos H, Kelly JJ, Cistulli PA. Oral appliance therapy reduces blood pressure in obstructive sleep apnea: a randomized, controlled trial. Sleep 2004;27:934-941.

54. Lam B, Sam K, Lam JC, Lai AY, Lam CL, Ip MS. The efficacy of oral appliances in the treatment of severe obstructive sleep apnea. Sleep Breath 2011;15:195-201.

55. Gauthier L, Laberge L, Beaudry M, Laforte M, Rompré PH, Lavigne GJ. Efficacy of two mandibular advancement appliances in the management of snoring and mild-moderate sleep apnea: a cross-over randomized study. Sleep Med 2009;10:329-336.

56. Trzepizur W, Gagnadoux F, Abraham P, et al. Microvascular endothelial function in obstructive sleep apnea: Impact of continuous positive airway pressure and mandibular advancement. Sleep Med 2009;10:746-752.

57. Itzhaki S, Dorchin H, Clark G, Lavie L, Lavie P, Pillar G. The effects of 1-year treatment with a herbst mandibular advancement splint on obstructive sleep apnea, oxidative stress, and endothe- 
lial function. Chest 2007;131:740-749.

58. Anandam A, Patil M, Akinnusi M, Jaoude P, El-Solh AA. Cardiovascular mortality in obstructive sleep apnoea treated with continuous positive airway pressure or oral appliance: an observational study. Respirology 2013;18:1184-1190.

59. Liu Y, Park YC, Lowe AA, Fleetham JA. Supine cephalometric analyses of an adjustable oral appliance used in the treatment of obstructive sleep apnea. Sleep Breath 2000;4:59-66.

60. Liu Y, Lowe AA, Fleetham JA, Park YC. Cephalometric and physiologic predictors of the efficacy of an adjustable oral appliance for treating obstructive sleep apnea. Am J Orthod Dentofacial Orthop 2001;120:639-647.

61. Hoekema A, Stegenga B, Wijkstra PJ, van der Hoeven JH, Meinesz AF, de Bont LG. Obstructive sleep apnea therapy. J Dent Res 2008;87:882-887.

62. Chung JW, Enciso R, Levendowski DJ, Morgan TD, Westbrook PR, Clark GT. Treatment outcomes of mandibular advancement devices in positional and nonpositional OSA patients. Oral Surg Oral Med Oral Pathol Oral Radiol Endod 2010;109:724-731.

63. Marklund M, Stenlund H, Franklin KA. Mandibular advancement devices in 630 men and women with obstructive sleep apnea and snoring: tolerability and predictors of treatment success. Chest 2004;125:1270-1278.

64. Ferguson KA, Ono T, Lowe AA, al-Majed S, Love LL, Fleetham JA. A short-term controlled trial of an adjustable oral appliance for the treatment of mild to moderate obstructive sleep apnoea. Thorax 1997;52:362-368.

65. Battagel JM, Johal A, L'Estrange PR, Croft CB, Kotecha B. Changes in airway and hyoid position in response to mandibular protrusion in subjects with obstructive sleep apnoea (OSA). Eur J Orthod 1999;21:363-376.

66. Popovic RM, White DP. Influence of gender on waking genioglossal electromyogram and upper airway resistance. Am J Respir Crit Care Med 1995;152:725-731.

67. Collop NA. The significance of sleep-disordered breathing and obstructive sleep apnea in the elderly. Chest 1997;112:867-868.

68. Sanner BM, Heise M, Knoben B, et al. MRI of the pharynx and treatment efficacy of a mandibular advancement device in obstructive sleep apnoea syndrome. Eur Respir J 2002;20:143-150.

69. Almeida FR, Lowe AA, Otsuka R, Fastlicht S, Farbood M, Tsuiki S. Long-term sequellae of oral appliance therapy in obstructive sleep apnea patients: Part 2. Study-model analysis. Am J Orthod Dentofacial Orthop 2006;129:205-213.

70. Marklund M. Predictors of long-term orthodontic side effects from mandibular advancement devices in patients with snoring and obstructive sleep apnea. Am J Orthod Dentofacial Orthop 2006;129:214-221.

71. de Almeida FR, Bittencourt LR, de Almeida CI, Tsuiki S, Lowe AA, Tufik S. Effects of mandibular posture on obstructive sleep apnea severity and the temporomandibular joint in patients fitted with an oral appliance. Sleep 2002;25:507-513.

72. Pliska BT, Almeida F. Effectiveness and outcome of oral appliance therapy. Dent Clin North Am 2012;56:433-444.

73. Pitsis AJ, Darendeliler MA, Gotsopoulos H, Petocz P, Cistulli PA.
Effect of vertical dimension on efficacy of oral appliance therapy in obstructive sleep apnea. Am J Respir Crit Care Med 2002;166: 860-864.

74. Nikolopoulou M, Naeije M, Aarab G, Hamburger HL, Visscher CM, Lobbezoo F. The effect of raising the bite without mandibular protrusion on obstructive sleep apnoea. J Oral Rehabil 2011;38: 643-647.

75. Kato J, Isono S, Tanaka A, et al. Dose-dependent effects of mandibular advancement on pharyngeal mechanics and nocturnal oxygenation in patients with sleep-disordered breathing. Chest 2000;117:1065-1072.

76. Aarab G, Lobbezoo F, Hamburger HL, Naeije M. Effects of an oral appliance with different mandibular protrusion positions at a constant vertical dimension on obstructive sleep apnea. Clin Oral Investig 2010;14:339-345.

77. Tegelberg A, Walker-Engström ML, Vestling 0, Wilhelmsson B. Two different degrees of mandibular advancement with a dental appliance in treatment of patients with mild to moderate obstructive sleep apnea. Acta Odontol Scand 2003;61:356-362.

78. Ahrens A, McGrath C, Hägg U. A systematic review of the efficacy of oral appliance design in the management of obstructive sleep apnoea. Eur J Orthod 2011;33:318-324.

79. Battagel JM, Kotecha B. Dental side-effects of mandibular advancement splint wear in patients who snore. Clin Otolaryngol 2005;30:149-156.

80. Marklund M, Sahlin C, Stenlund H, Persson M, Franklin KA. Mandibular advancement device in patients with obstructive sleep apnea: long-term effects on apnea and sleep. Chest 2001; 120:162-169.

81. Lee WH, Wee JH, Lee CH, et al. Comparison between mono-bloc and bi-bloc mandibular advancement devices for obstructive sleep apnea. Eur Arch Otorhinolaryngol 2013;270:2909-2913.

82. Rose E, Staats R, Virchow C, Jonas IE. A comparative study of two mandibular advancement appliances for the treatment of obstructive sleep apnoea. Eur J Orthod 2002;24:191-198.

83. Ghazal A, Sorichter S, Jonas I, Rose EC. A randomized prospective long-term study of two oral appliances for sleep apnoea treatment. J Sleep Res 2009;18:321-328.

84. Vanderveken OM, Devolder A, Marklund M, et al. Comparison of a custom-made and a thermoplastic oral appliance for the treatment of mild sleep apnea. Am J Respir Crit Care Med 2008;178:197-202.

85. Tan YK, L'Estrange PR, Luo YM, et al. Mandibular advancement splints and continuous positive airway pressure in patients with obstructive sleep apnoea: a randomized cross-over trial. Eur J Orthod 2002;24:239-249.

86. Almeida FR, Henrich N, Marra C, et al. Patient preferences and experiences of CPAP and oral appliances for the treatment of obstructive sleep apnea: a qualitative analysis. Sleep Breath 2013; 17:659-666.

87. Giles TL, Lasserson TJ, Smith BJ, White J, Wright J, Cates CJ. Continuous positive airways pressure for obstructive sleep apnoea in adults. Cochrane Database Syst Rev 2006;(1):CD001106. 